\title{
Neighborhood Socioeconomic Disparities and 1-year Case Fatality after Incident Myocardial Infarction: The Atherosclerosis Risk in Communities (ARIC) Community Surveillance (1992- 2002)
}

\author{
Randi E. Foraker, Ph.D. ${ }^{1}$, Mehul D. Patel, MSPH $^{2}$, Eric A. Whitsel, MD, MPH $^{2}$, Chirayath M. \\ Suchindran, Ph.D. ${ }^{3}$, Gerardo Heiss, MD, Ph.D. ${ }^{2}$, and Kathryn M. Rose, Ph.D. \\ ${ }^{1}$ Division of Epidemiology, The Ohio State University, 1841 Neil Avenue, Columbus, $\mathrm{OH}, 43210$, \\ USA \\ 2Department of Epidemiology, University of North Carolina at Chapel Hill, 135 Dauer Drive, \\ Chapel Hill, NC, 27599, USA \\ ${ }^{3}$ Department of Biostatistics, University of North Carolina at Chapel Hill, 135 Dauer Drive, Chapel \\ Hill, NC, 27599, USA
}

${ }^{4}$ SRA International, 2605 Meridian Parkway, Durham, NC, 27713, USA

\section{Abstract}

\begin{abstract}
Background-Declines in case-fatality post-myocardial infarction (MI) have been observed over the past three decades. Few studies report socioeconomic disparities in survival post-MI.

Methods-We assessed 1-year case fatality among 9,116 incident MI patients included in the Atherosclerosis Risk in Communities (ARIC) community surveillance from 1992 to 2002.

Addresses of hospitalized MI patients were geocoded by a commercial vendor and linked to year 2000 United States (US) Census tract-level neighborhood income (nINC) data. We estimated case fatality odds ratios and $95 \%$ confidence intervals (OR, 95\% CI) with a multinomial logistic model to quantify the association between nINC tertile and case fatality, comparing short- (within 28 days) and long-term (29 to 365 days) case fatality to no death 1-year post-MI.
\end{abstract}

Results-Overall, 1-year age-adjusted case fatality rates were highest among MI patients living in low nINC areas, followed by medium and high nINC areas, respectively. We found significant OR modification by race $(\mathrm{p}<0.10)$, and formed race-nINC strata with high nINC whites as the referent group. In multivariable models, adjusting for age, gender, study community, and year of MI, low nINC whites and low and medium nINC blacks had higher short-term case fatality than high nINC whites. Low and medium nINC blacks had higher long-term case fatality compared to high nINC whites.

Conclusions-Differences in short- and long-term case fatality by neighborhood socioeconomic factors have not been systematically studied in the US. Surveillance efforts can be expanded to incorporate measures of the neighborhood context to examine these associations over time.

\footnotetext{
(C) 2012 Mosby, Inc. All rights reserved.

Corresponding author: Randi E. Foraker, The Ohio State University College of Public Health, 334 Cunz Hall | 1841 Neil Avenue, Columbus, OH 43210-3533, 614-292-9916 (office), 614-688-3533 (fax), rforaker@ cph.osu.edu.

Publisher's Disclaimer: This is a PDF file of an unedited manuscript that has been accepted for publication. As a service to our customers we are providing this early version of the manuscript. The manuscript will undergo copyediting, typesetting, and review of the resulting proof before it is published in its final citable form. Please note that during the production process errors may be discovered which could affect the content, and all legal disclaimers that apply to the journal pertain.
} 


\section{INTRODUCTION}

Among United States (US) Medicare beneficiaries, declines in case fatality post-myocardial infarction (MI) were observed between 1984 and 2003. ${ }^{1}$ Declines were greatest for whites compared to blacks, and the racial disparities in case fatality were more evident for longterm (1-year) than for short-term (30-day) case-fatality. ${ }^{1}$ Data from Norway suggest lower short- and long-term case fatality among women compared to men, ${ }^{2}$ while data from Sweden demonstrate post-MI survival differences by gender vary by age. ${ }^{3}$

While socioeconomic disparities in coronary heart disease (CHD) are well-documented in US cohort studies, ${ }^{4-7}$ relatively few studies have focused on socioeconomic disparities in short- and long-term survival among those who initially survive an MI. One study from Scotland showed little evidence of area-level socioeconomic inequality in 28-day case fatality, ${ }^{8}$ while other studies from Northern Europe found that 28 -day ${ }^{9}, 10$ and 1-year ${ }^{11}$ case fatality was higher, and survival time lower ${ }^{12}$, among those from low versus high socioeconomic status (SES) areas.

Socioeconomic disparities in survival are less systematically studied in the US, as socioeconomic data are not generally available in administrative databases and hospital records. However, results from the Worcester Heart Attack Study ${ }^{13}$ and a cohort of elderly Medicare beneficiaries living in Chicago ${ }^{14}$ suggest that lower neighborhood-level SES is associated with shorter survival post-MI over a follow-up period of 6 or 7 years. Although socioeconomic disparities appear to exist in short- and long-term case fatality post-MI, the role of socioeconomic differences in biracial populations has not been sufficiently evaluated. There exist considerable public health implications for including socioeconomic data in ongoing surveillance of MI case fatality.

We therefore examined the association between neighborhood-level median household income (nINC) and 1-year case fatality after an incident hospitalized MI, and determined the extent to which case fatality differed by age, race/ethnicity, gender, and year of MI. We also investigated the association of nINC with short-term ( ㅅ8-day) and longer-term (29-day to 1 -year) case fatality, and then quantified the influence of other patient-level and hospitallevel factors on these measures.

\section{METHODS}

The Atherosclerosis Risk in Communities (ARIC) community surveillance has been ongoing since 1987, and its methods have been described. ${ }^{15}$ Briefly, both hospitalized and fatal CHD events are systematically identified among residents aged 35-74 years from 4 US communities: Washington County, Maryland (MD); northwest suburbs of Minneapolis, Minnesota (MN); Jackson, Mississippi (MS); and Forsyth County, North Carolina (NC).

Selected ICD-9 codes $(402,410-414,427,428,518.4)$ were sampled yearly from listings of discharge diagnoses from hospitals in each of the 4 geographically specified communities. We defined definite and probable myocardial infarction (MI) by standardized algorithm using information from electrocardiograms, cardiac symptoms (pain), and biomarker concentrations. We included all definite and probable MI events sampled from 1992 to 2002 $(\mathrm{n}=10,360)$. We excluded 2,312 events with a history of MI or CHD; 1,080 residing outside of the study area; 484 with non-white race, younger than 35 or older than 74 years of age, or missing sampling weight. After exclusions, the unweighted number of events was 6,484. Upon weighting for the ICD-9 sampling strategy, there were 9,116 incident hospitalized MI events available for analysis. 
Addresses of hospitalized incident MI patients were geocoded by a commercial vendor selected on the basis of accuracy of geocoding ${ }^{16}$ and linked to year 2000 US Census tractlevel socioeconomic data. Of these addresses, $93 \%$ were exact address matches, and an additional $2 \%$ were tract centroid matches. ${ }^{7}$ We categorized tract-level nINC across the 4 study communities into tertiles: low $(<\$ 33,533)$; medium $(\$ 33,533-50,031)$; and high (> $\$ 50,031)$.

Detailed methods for ascertaining and classifying fatal events for surveillance investigation are described elsewhere. ${ }^{15}$ Deaths occurring within 1 year of discharge among patients hospitalized with MI in ARIC community surveillance are recorded from death certificate data or ascertained from National Death Index (NDI) searches. We defined 1-year case fatality as death occurring within 1 year of the hospital discharge for an incident MI. Within 1 -year case fatality, we further classified longer-term case fatality as occurring between 29 and 365 days, and short-term case fatality as death within 28 days.

We estimated case fatality odds ratios and 95\% confidence intervals (OR, 95\% CI) with a multinomial logistic model using generalized estimation equations with robust variance estimators to account for the clustering of patients within census tracts. We used multivariate modeling, weighted to account for the sampling by hospital discharge codes, to estimate the association between nINC tertile and short-term MI case fatality ( 28 days) and long-term MI case fatality (29 to 365 days) compared to no death 1-year post-MI (SAScallable SUDAAN, release 9.0.1; Research Triangle Institute, Research Triangle Park, NC).

We accounted for the following covariates in statistical models of the nINC-case fatality association: age, race (black or white), gender, study community, year of MI, health insurance type (private, Medicare, Medicaid, no insurance), hospital type (teaching, nonteaching), and medical history (diabetes, hypertension, heart failure, stroke, angina, and current smoking). We additionally assessed for modification of the odds ratio by race $(\mathrm{p}<0.10)$. Initial multivariable analyses showed a significant race-nINC interaction $(\mathrm{p}=0.08)$. For subsequent race-specific estimates, we used data from the 2 biracial communities. Multinomial modeling increased power for detecting differences in case fatality between race-nINC groups.

This research was funded in part by the National Institutes of Health, National Heart, Lung, and Blood Institute, and National Research Service Award training grant. The authors are solely responsible for the design and conduct of this study, all study analyses, the drafting and editing of the paper, and its final contents.

\section{RESULTS}

US Census (2000) characteristics of the communities in which eligible hospitalized incident MI cases resided are described in Table 1. Whites comprised the majority of the MD and MN communities, while blacks were more highly represented in the biracial communities of Jackson, MS and Forsyth County, NC. Among 9,116 incident MI cases hospitalized from 1992-2002 in 4 ARIC study communities, we observed a greater proportion of females, blacks, and Medicaid recipients living in low nINC areas compared to higher nINC areas (Table 2). MI patients living in low nINC areas also had a higher prevalence of all measured clinical comorbidities compared to MI patients living in higher nINC areas (Table 2).

Overall, 1-year age-adjusted case fatality rates across all 4 study communities were highest among MI patients living in low nINC areas, followed by medium and high nINC areas, respectively. When we stratified 1-year case fatality rates by long-term (29 day to 1 year) and short-term ( 28 days) case fatality, the inverse relationship of case fatality with nINC held for longer-term case fatality, but not for short-term case fatality (Figure 1). However, a 
significantly higher short-term case fatality among MI patients living in low nINC areas compared to those living in higher nINC areas persisted (Figure 1).

We also observed differences in the relationship between nINC and 1-year age-adjusted case fatality rates by race. Across all 4 study communities, 1-year case fatality rates decreased with increasing nINC for whites. Among black MI patients, those living in low and medium nINC areas had similar case fatality, which was significantly higher compared to those living in high nINC areas (Figure 2). In addition, blacks had consistently higher 1-year postMI case fatality rates than white MI patients living in low and medium nINC areas, but not high nINC areas (Figure 2). A clear nINC-case fatality gradient was not apparent for blacks or whites in longer-term or short-term case fatality strata. However, we observed that estimates of longer-term case fatality were larger for blacks living in lower nINC areas compared to whites living in lower nINC areas (Figure 2).

Estimates of short-term case fatality did not differ by race, but there was a trend toward higher 28-day case fatality among MI patients living in low nINC areas compared to those living in high nINC areas (Figure 2). Imprecision in the 1-year case fatality estimates for certain nINC/race groups can be seen in the width of the $95 \%$ confidence intervals in Figure 2. For example, there are relatively fewer medium and high nINC blacks, and low nINC whites, compared to the other nINC/race groups.

We found significant effect modification of the nINC-case fatality association by race $(\mathrm{p}=0.08)$. Thus, we assessed the nINC-case fatality relationship among blacks and whites from the 2 biracial study communities (MS and NC). We formed race-nINC strata, and high $\mathrm{nINC}$ whites served as the referent group. In multivariable models, adjusting for age, gender, study community, and year of MI, low nINC whites and both low and medium nINC blacks had higher 28-day case fatality rates compared to high nINC whites. In the same model, low and medium nINC blacks had higher longer-term case fatality rates compared to high nINC whites (Figure 3; Table 3).

We incorporated hospital teaching status into the model shown in Figure 3, and while the results were attenuated, the effect of hospital teaching status did not substantially change our results. We added type of health insurance to the model shown in Figure 3 (data not shown), and significantly higher case fatality rates persisted among low and medium nINC blacks, compared to high nINC whites for both longer- and short-term case fatality periods.

Meanwhile, higher 28-day case fatality rates were no longer seen among low versus high nINC whites.

Finally, differences in both short- and longer-term case fatality observed between low nINC blacks and high nINC whites (Figure 3) were robust after additional adjustment for history of diabetes, hypertension, heart failure, stroke, angina, and current smoking (Table 3). While blacks living in medium nINC areas experienced higher longer-term case fatality compared to high nINC whites, the elevated short-term case fatality rates seen among low nINC whites compared to high nINC whites did not persist with inclusion of medical history variables (Table 3).

\section{DISCUSSION}

In this community-based investigation of mortality following a hospitalization for a first MI, residence in low-income neighborhoods was associated with elevated 1-year MI case fatality. In the biracial communities, when compared to high nINC whites, 1-year case fatality was significantly higher for blacks living in low and medium nINC areas. Meanwhile, residence in low-income neighborhoods was associated with higher short- and long-term case fatality compared to residence in high-income neighborhoods. In addition, 
both short- and long-term case fatality was higher among blacks living in low-income neighborhoods compared to whites living in low-income neighborhoods.

nINC associations with case fatality were not entirely explained in this study by individual differences in health insurance status, hospital teaching status, or medical history variables. These results are consistent with other published studies of short- and long-term MI case fatality. ${ }^{9-14}$ Given the nature of these surveillance data, we were unable to identify pathways involved in the nINC-case fatality association. Our findings suggest that the influence of nINC, independent of individual-level factors, on MI case fatality may be due to access to preventive health care and treatments, level of environmental stressors, amount of social support among neighbors, and a health-oriented built environment that in turn may have important implications for self-care practices. It is notable that there exists a large, yet statistically insignificant, difference in long-term case fatality between high nINC blacks and whites. It remains unknown if unmeasured factors, such as individual-level SES or the neighborhood environment, differ between blacks and whites in high nINC settings. Regardless, findings from other studies suggest an independent effect of neighborhood SES on MI case fatality after controlling for individual-level SES. ${ }^{11,12,14}$

We observed nINC associations of larger magnitude for longer-term compared to short-term case fatality for blacks. This finding suggests that, among blacks, the effect of nINC may play a larger role in issues related to long-term post-MI treatment, such as compliance with treatment, access to primary care, and the quality of medical care received, such as evidence-based pharmacotherapy; ${ }^{17}$ however, data are not available that allow us to address these potential mechanisms in our work. ${ }^{18}$ In contrast, factors such as disease severity may be more strongly linking nINC to short-term case fatality, as more severe disease and a higher burden of comorbidity often leads to shorter time to death.

A strength of the current study is the ongoing community surveillance by ARIC with its comprehensive assessment of hospitalized MI events in the community and its standardized classification of hospitalized events. The ability to ascertain mortality data following an incident MI hospitalization across the 4 ARIC study communities further strengthens the characterization of long- and short-term case fatality. The high geocoding match rate to the level of the tract is an additional strength of these data. A limitation of these analyses is that we defined patient neighborhoods by their tract of residence. While tracts are administrative units designed to be socio-demographically homogenous, it is likely that the patients' perception of their neighborhood does not follow tract boundaries.

Further, the 2 ARIC surveillance communities with substantial black populations are in the southern US, and may not be representative of populations in other regions of the country. However, the magnitude of variation in $\mathrm{nINC}$ by race seen in these communities in our previous work ${ }^{19}$ was comparable to national estimates. ${ }^{20}$ As there were relatively few blacks living in higher nINC areas and few whites living in low nINC areas, our case fatality estimates for these race/nINC groups were less precise. In addition, at the time these surveillance data were collected, the upper age limit was 74 years. More contemporary surveillance data include those aged 35 to 84 years, allowing for inference to the very old.

Although racial disparities in MI case fatality have been previously documented, ${ }^{1,21,} 22$ differences in long- and short-term case fatality by neighborhood socioeconomic factors have not been systematically studied in the US. Since address data are readily available in medical records, and patients' addresses can be geocoded to determine place of residence at the time of hospitalization, CHD surveillance efforts can be expanded to incorporate measures of the neighborhood context in order to examine socioeconomic disparities in long- and short-term MI case fatality over time. 


\section{Acknowledgments}

This research was supported by contract 1R01HL080287 (Dr. Rose, PI) from the National Heart, Lung, and Blood Institute (NHLBI) and was also funded in part by National Institutes of Health, NHLBI, and National Research Service Award training grant 5-T32-HL007055. The ARIC study is carried out as a collaborative study supported by NHLBI contracts N01-HC-55015, N01-HC-55016, N01-HC-55018, N01-HC-55019, N01-HC-55020, N01HC-55021, and N01-HC-55022. We thank the staff of the ARIC study for their important contributions.

\section{References}

1. Wellenius GA, Mittleman MA. Disparities in myocardial infarction case fatality rates among the elderly: The 20-year MSedicare experience. American Heart Journal. 2008; 156:483-490. [PubMed: 18760130]

2. Langørgen J, Igland J, Vollset SE, et al. Short-term and long-term case fatality in 11878 patients hospitalized with a first acute myocardial infarction, 1979-2001: The western Norway cardiovascular registry. Journal of Cardiovascular Risk. 2009; 16:621-627.

3. Rosengren A, Spetz C-L, Köster M, et al. Sex differences in survival after myocardial infarction in sweden. Data from the Swedish national acute myocardial infarction register. European Heart Journal. 2001; 22:314-322. [PubMed: 11161950]

4. Diez Roux AV, Merkin SS, Arnett D, et al. Neighborhood of residence and incidence of coronary heart disease. N Engl J Med. 2001; 345:99-106. [PubMed: 11450679]

5. Diez-Roux AV, Nieto FJ, Muntaner C, et al. Neighborhood environments and coronary heart disease: A multilevel analysis. Am J Epidemiol. 1997; 146:48-63. [PubMed: 9215223]

6. LeClere FB, Rogers RG, Peters K. Neighborhood social context and racial differences in women's heart disease mortality. J Health Soc Behav. 1998; 39:91-107. [PubMed: 9642901]

7. Rose KM, Suchindran CM, Foraker RE, et al. Neighborhood disparities in incident hospitalized myocardial infarction in four U.S. Communities: The ARIC surveillance study. Annals of Epidemiology. 2009; 19:867-874. [PubMed: 19815428]

8. Davies C, Leyland A. Trends and inequalities in short-term acute myocardial infarction case fatality in Scotland, 1988-2004. Popul Health Metr. 2010; 6:33. [PubMed: 21134255]

9. Gerward S, Tyden P, Hansen O, et al. Survival rate 28 days after hospital admission with first myocardial infarction. Inverse relationship with socioeconomic circumstances. Journal of Internal Medicine. 2006; 259:164-172. [PubMed: 16420545]

10. Morrison C, Woodward M, Leslie W, et al. Effect of socioeconomic group on incidence of, management of, and survival after myocardial infarction and coronary death: Analysis of community coronary event register. BMJ. 1997; 314:541-546. [PubMed: 9055711]

11. Winkleby M, Sundquist K, Cubbin C. Inequities in CHD incidence and case fatality by neighborhood deprivation. American Journal of Preventive Medicine. 2007; 32:97-106. [PubMed: 17234484]

12. Chaix B, Rosvall M, Merlo J. Neighborhood socioeconomic deprivation and residential instability: Effects on incidence of ischemic heart disease and survival after myocardial infarction. Epidemiology. 2007; 18:104-111. [PubMed: 17130687]

13. Tonne C, Schwartz J, Mittleman M, et al. Long-term survival after acute myocardial infarction is lower in more deprived neighborhoods. Circulation. 2005; 111:3063-3070. [PubMed: 15939820]

14. Wen M, Christakis NA. Neighborhood effects on posthospitalization mortality: A population-based cohort study of the elderly in chicago. Health Services Research. 2005; 40:1108-1127. [PubMed: 16033495]

15. White AD, Folsom AR, Chambless LE, et al. Community surveillance of coronary heart disease in the Atherosclerosis Risk in Communities (ARIC) study: Methods and initial two years' experience. J Clin Epidemiol. 1996; 49:223-233. [PubMed: 8606324]

16. Whitsel EA, Rose KM, Wood JL, et al. Accuracy and repeatability of commercial geocoding. Am J Epidemiol. 2004; 160:1023-1029. [PubMed: 15522859]

17. Foraker R, Rose K, Whitsel E, et al. Neighborhood socioeconomic status, medicaid coverage and medical management of myocardial infarction: Atherosclerosis Risk in Communities (ARIC) community surveillance. BMC Public Health. 2010; 10:632. [PubMed: 20964853] 
18. Kaufman JS. Epidemiologic analysis of racial/ethnic disparities: Some fundamental issues and a cautionary example. Social Science \& Medicine. 2008; 66:1659-1669. [PubMed: 18248866]

19. Foraker RE, Rose KM, Kucharska-Newton AM, et al. Variation in rates of fatal coronary heart disease by neighborhood socioeconomic status: The Atherosclerosis Risk in Communities surveillance (1992-2002). Annals of Epidemiology. 2011; 21:580-588. [PubMed: 21524592]

20. Bavas-Walt C, Proctor BD, Mills R. Income, poverty, and health insurance coverage in the United States. 2003

21. Galea S, Blaney S, Nandi A, et al. Explaining racial disparities in incidence of and survival from out-of-hospital cardiac arrest. Am J Epidemiol. 2007; 166:534-543. [PubMed: 17584756]

22. Kostis JB, Wilson AC, Lacy CR, et al. Time trends in the occurrence and outcome of acute myocardial infarction and coronary heart disease death between 1986 and 1996 (a new jersey statewide study). Am J Cardiol. 2001; 88:651-652. [PubMed: 11564389] 


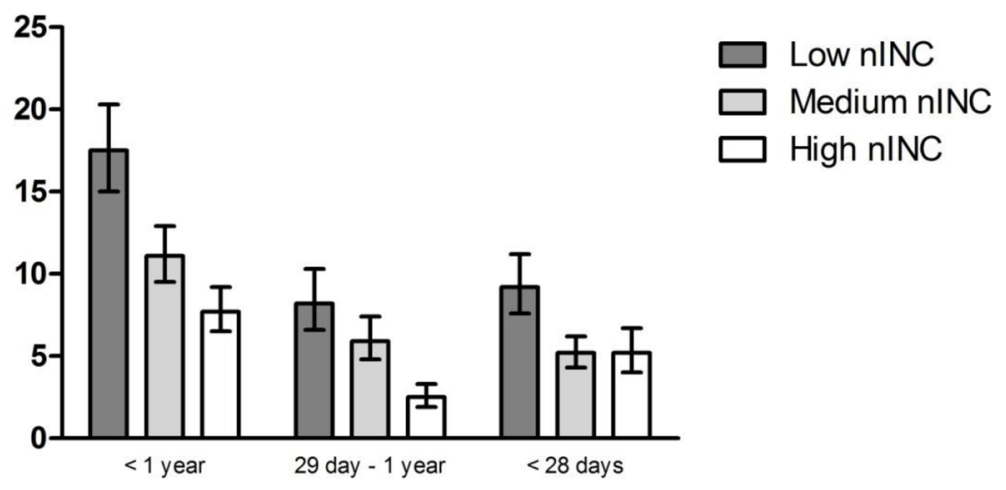

Figure 1.

Age-adjusted 1-year, long- and short-term case fatality (percent and 95\% confidence limits) by nINC among incident MI cases: ARIC community surveillance (1992-2002) 


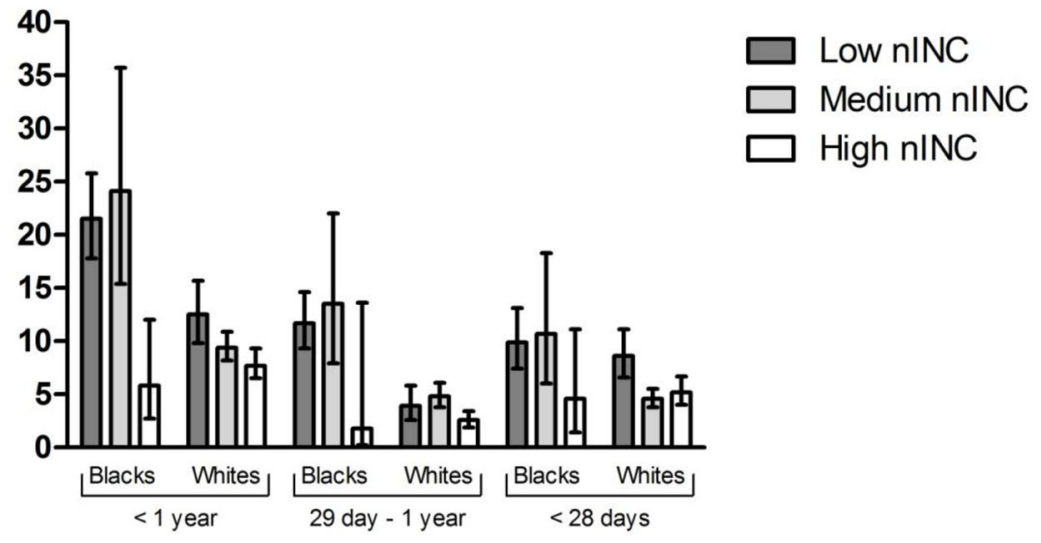

Figure 2.

Age-adjusted case fatality (percent and $95 \%$ confidence limits) by nINC among incident MI cases: ARIC community surveillance (1992-2002), blacks and whites 


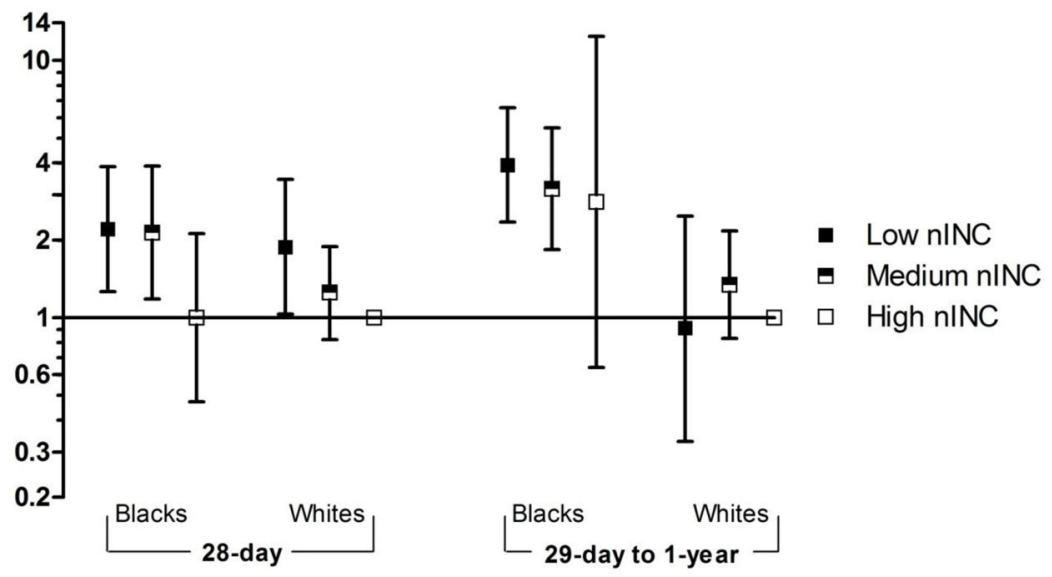

Figure 3.

Case fatality odds ratio (OR) and 95\% CI by race-nINC groups among incident MI cases: ARIC community surveillance; Forsyth County, NC and Jackson, MS (1992-2002)

Adjusted for age, gender, study community, and year of MI 
Table 1

Characteristics (N) of Eligible Population by ARIC Study Community, 2000 Census

\begin{tabular}{|c|c|c|c|c|}
\hline & Washington Co. Maryland & Minneapolis Minnesota & Jackson (city) Mississippi & $\begin{array}{l}\text { Forsyth Co. North } \\
\text { Carolina }\end{array}$ \\
\hline \multicolumn{5}{|l|}{ Race-gender group } \\
\hline Black Women & 1,330 & 4,694 & 26,976 & 18,181 \\
\hline Black Men & 1,220 & 4,380 & 21,545 & 15,175 \\
\hline White Women & 29,048 & 48,329 & 8,491 & 53,272 \\
\hline White Men & 27,033 & 45,168 & 7,137 & 47,887 \\
\hline Total population * & 58,631 & 102,571 & 64,149 & 134,515 \\
\hline Census tracts & 31 & 55 & 43 & 75 \\
\hline $\begin{array}{l}\text { Average persons per census } \\
\text { tract }\end{array}$ & 1,891 & 1,865 & 1,492 & 1,794 \\
\hline
\end{tabular}


Table 2

Characteristics (\%) of incident MI cases by nINC: ARIC community surveillance (1992-2002)

\begin{tabular}{|c|c|c|c|}
\hline & Low nINC n=2,981 & Medium nINC n=3,398 & High nINC n=2,737 \\
\hline Age [mean (SD)] & $58.9(13.0)$ & $60.2(10.9)$ & $59.9(11.2)$ \\
\hline Female $(\%)$ & 44.0 & 34.1 & 30.3 \\
\hline Black & 59.1 & 11.3 & 4.1 \\
\hline \multicolumn{4}{|l|}{ Study community } \\
\hline $\mathrm{NC}$ & 34.5 & 47.3 & 34.1 \\
\hline MS & 53.8 & 12.5 & 14.9 \\
\hline MN & 1.3 & 15.0 & 42.3 \\
\hline MD & 10.5 & 25.2 & 8.6 \\
\hline \multicolumn{4}{|l|}{ Year of event } \\
\hline 1992-1995 & 37.5 & 33.4 & 31.5 \\
\hline 1996-1998 & 30.1 & 31.6 & 27.3 \\
\hline 1999-2002 & 32.5 & 34.9 & 41.2 \\
\hline \multicolumn{4}{|l|}{ Health insurance } \\
\hline Prepaid and/or Medicare & 63.7 & 86.3 & 91.2 \\
\hline Medicaid & 18.8 & 4.5 & 2.8 \\
\hline Other & 4.0 & 1.7 & 1.9 \\
\hline No insurance & 13.5 & 7.6 & 4.2 \\
\hline Teaching hospital & 34.9 & 33.2 & 49.5 \\
\hline \multicolumn{4}{|l|}{ Medical history } \\
\hline Current smoker & 45.6 & 42.4 & 37.8 \\
\hline Hypertension & 71.0 & 56.7 & 51.9 \\
\hline Diabetes & 38.5 & 27.6 & 23.3 \\
\hline $\mathrm{CHF}$ & 38.3 & 31.9 & 25.4 \\
\hline Angina & 21.8 & 18.3 & 20.7 \\
\hline Stroke & 11.2 & 6.8 & 6.0 \\
\hline
\end{tabular}


Table 3

Case fatality odds ratio (OR) and 95\% CI by race-nINC groups among incident MI cases: ARIC community surveillance; Forsyth County, NC and Jackson, MS (1992-2002)

\begin{tabular}{lllll}
\hline & \multicolumn{2}{c}{ Model 1* } & \multicolumn{2}{c}{ Model 2 $^{\dagger}$} \\
& s8 days & 29 days-1 year & ş8 days & 29 days-1 year \\
\hline Low nINC black & $2.21(1.26,3.86)$ & $3.92(2.35,6.55)$ & $2.07(1.06,4.02)$ & $2.82(1.46,5.44)$ \\
Medium nINC black & $2.14(1.18,3.88)$ & $3.17(1.84,5.47)$ & $1.73(0.90,3.34)$ & $1.94(1.03,3.66)$ \\
High nINC black & $1.00(0.47,2.12)$ & $2.82(0.64,12.4)$ & $1.02(0.45,2.32)$ & $3.15(0.72,13.8)$ \\
Low nINC white & $1.88(1.02,3.45)$ & $0.91(0.33,2.48)$ & $1.62(0.84,3.13)$ & $0.94(0.36,2.48)$ \\
Medium nINC white & $1.25(0.82,1.89)$ & $1.34(0.83,2.17)$ & $1.06(0.68,1.67)$ & $1.15(0.68,1.96)$ \\
High nINC white & 1.00 (referent) & 1.00 (referent) & 1.00 (referent) & 1.00 (referent) \\
\hline
\end{tabular}

* Model 1 adjusted for age, gender, study community, and year of MI

${ }^{\dagger}$ Model 2 adjusted for factors in Model 1, plus history of diabetes, hypertension, heart failure, stroke, angina, and current smoking 